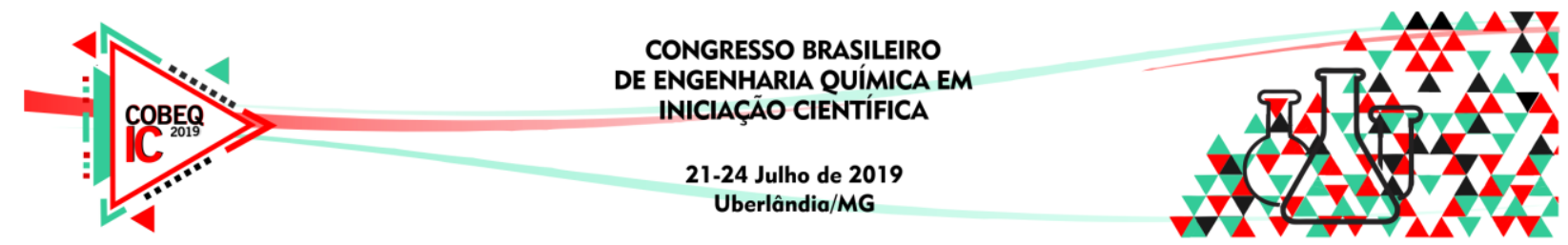

\title{
EFEITO DA VARIAÇÃO DA TEMPERATURA E ÂNGULO DE LIGAÇÃO DA AMILOPECTINA EM PRESENÇA DE ÁGUA POR MODELAGEM MOLECULAR
}

\author{
J. P. O. LIMA ${ }^{1}$, E. MOCHIUTTI ${ }^{1}$, A. L. S. CARVALHO ${ }^{1}$, A. E. S. NASCIMENTO ${ }^{1}$, M. C. \\ MARTELLI ${ }^{1}$ \\ ${ }^{1}$ Universidade Federal do Pará, Faculdade de Engenharia Química \\ E-mail para contato: pedroolima@live.com
}

\begin{abstract}
RESUMO - Este trabalho visa a aplicação de simulação computacional para o estudo da estrutura da amilopectina torcionada e solvatada em água, com intuito de analisar a influência da variação da temperatura na energia do sistema. Para a construção da estrutura da amilopectina utilizou-se o software Glycam carbohydrate builder, bem como o auxílio do software Spartan Wavefunction V.8 e do servidor Automated Topology Builder (ATB) para o cálculo de minimização de energia e análise conformacional. Após a otimização foi utilizado o software GROMACS para a realização da dinâmica molecular. Conforme os resultados obtidos, observou-se que há uma relação de influência direta da temperatura com a variação de energia do sistema.
\end{abstract}

\section{INTRODUÇÃO}

$\mathrm{Na}$ atualidade, o acúmulo de materiais sintéticos não-biodegradáveis representa uma grande problemática para a conservação do meio ambiente. Um dos principais fatores para isto é o crescimento econômico e populacional, sendo estes relacionados de forma intrínseca com a produção desenfreada de produtos plásticos destinados a manufatura de embalagens. $\mathrm{O}$ principal fator atrativo da produção de produtos sintéticos plásticos se dá devido à ampla aplicabilidade e da sua alta durabilidade. Por consequência, a pesquisa e aplicação de polímeros biodegradáveis representa uma possível solução para a redução de impactos ao meio ambiente (Liporacci et al., 2005).

Nesse cenário, uma das principais formas para a redução de impactos ambientais é a produção de biofilmes, os quais são preparados a partir de materiais biológicos, comportandose como uma barreira para agentes externos. A fabricação de biofilmes envolve essencialmente a presença de três constituintes: um agente formador do filme, um solvente e um agente plastificante (Rocha et al., 2014).

Estudos como os de Lourdin et al. (1997) e Thiré et al. (2004) mostram que o amido é um polímero natural de grande potencial como agente formador do filme. O amido é composto por dois tipos de cadeias poliméricas de glucose, denominadas de amilose e amilopectina. Sendo um dos principais biopolímeros, encontrado facilmente na natureza em vegetais, frutas e legumes. A partir desta informação, o presente estudo tem como base a 


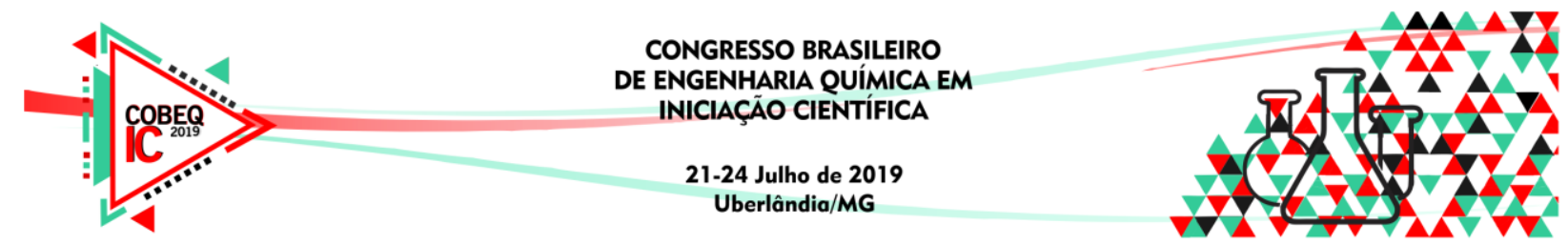

composição geral do amido, o qual é composto por amilose e amilopectina (Liporacci et al., 2005; Rocha et al., 2014).

Este trabalho tem como objetivo o estudo da forma estrutural da amilopectina, realizando análises para conformações com torções distintas, avaliando a energia de minimização destas. Por conseguinte, a amilopectina que apresentou menor conformação energética, foi solvatada em água, para a aplicação de dinâmica molecular no sistema (amilopectina e água), com intúito de estudar-se o efeito da variação de temperatura nos calculos de energia cinética, potencial e total.

\section{MATERIAIS E MÉTODOS}

\subsection{Construção da molécula}

A amilopectina é um polímero altamente ramificado, formado por cadeias de $\alpha$-Dglucose unidas através de ligações $\alpha-(1-4)$ e com ramificações de $4 \%$ a $6 \%$, unidas através de ligações $\alpha-(1-6)$ (Lajolo e Menezes, 2006). Seguindo as especificações descritas por Lajolo e Menezes (2006), foi construída uma molécula de amilopectina de fórmula molecular $\mathrm{C}_{66} \mathrm{H}_{112} \mathrm{O}_{56}$, composta de onze anéis de $\alpha$-D-glucose, de forma que, a cadeia principal possui nove anéis de $\alpha$-D-glucose (totalizando oito ligações $\alpha$-(1-4) entre estes aneis) e ramificação, a qual inicia no anel central da cadeia principal, composta de dois anéis, sendo o primeiro contendo uma ligação $\alpha-(1-6)$. O modelo desenvolvido da estrutura geral do polímero foi baseado no apresentado por Lajolo e Menezes (2006). A construção da estrutura modificada foi realizada através do software Glycam carbohydrate builder. Após a representação deste modelo foram aplicadas torções entre os anéis com graus variando de $180^{\circ}$ a $70^{\circ}$ e passo de $5^{\circ}$ entre cada estrutura. A estrutura geral estudada da amilopectina encontra-se demonstrada na Figura 1.

Figura 1 - Estrutura da amilopectina composta de 11 anéis $\alpha$-D-glucose.

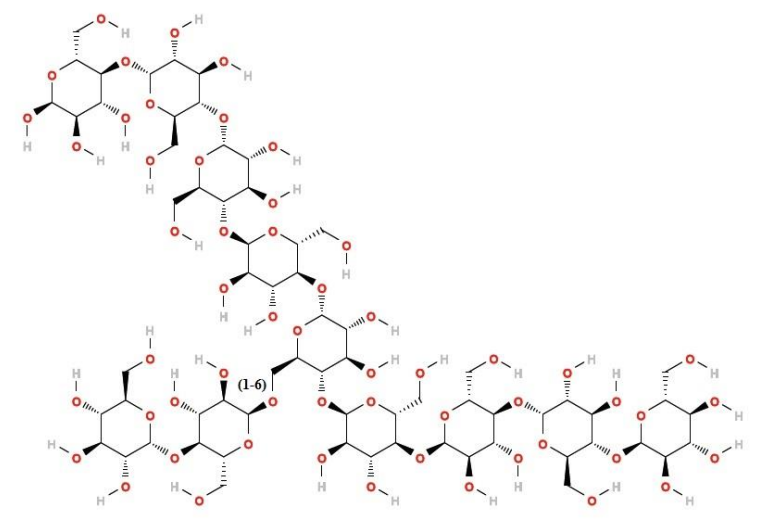

\subsection{Dinâmica molecular}

Utilizando o software Spartan Wavefunction V.8, através da aplicação do método semiempírico com base PM6, realizou-se o cálculo de minimização de energia para diversas conformações construídas. 


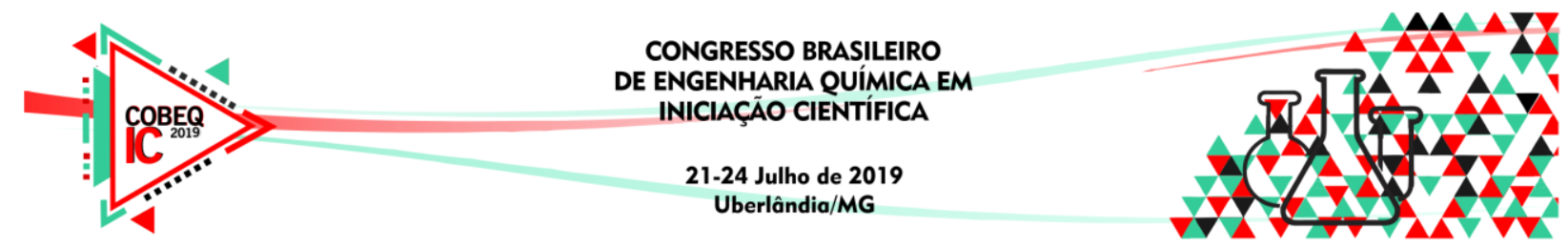

Após a realização dos cálculos de minimização de energia estrutural da amilopectina, selecionou-se a conformação de menor energia para uma otimização através do servidor Automated Topology Builder (ATB), onde executou-se um novo cálculo de minimização de energia, para a estrutura selecionada com o método Semi-Empírico.

Os cálculos de dinâmica molecular foram realizados utilizando o campo de força GROMOS 54a7 (Schmid et al.,2011). Foi criada uma caixa de solvatação com 11.154 moléculas de água, utilizando o modelo de carga pontual simples (SPC) descrito por Berendsen et al. (1981).

Primeiramente, realizou-se cálculos de minimização de energia, aplicando-se o método de máximo declive (steepest descent minimization), com um passo de energia de 0,01 e número total de passos de 50.000. Posteriormente, realizou-se duas etapas de equilíbrio, ambas com um tempo total de 250 ps cada. Uma etapa utilizando o conjunto NVT (número de mols, temperatura e volume constantes) e outra utilizando o conjunto NPT (número de mols, temperatura e pressão constantes). Em seguida, realizou-se o cálculo de dinâmica molecular com um número total de 500.000 passos, com um tempo de 1 ns. Para todas as simulações, utilizou-se condições periódicas de contorno em 3-Dimensões, o algoritmo de integração Leap-Frog, o algoritmo LINCS para a restrição do comprimento de todas as ligações. Para o cálculo de interações de longa distância, utilizou-se o método PME (Particle Mesh Ewald) com um corte de 1,4 nm. Para o controle de pressão, foi utilizado o barostato ParrinelloRahman, com uma escala isotrópica e pressão de referência de 1 bar. As simulações foram executadas em três temperaturas diferentes, sendo estas de $298,15 \mathrm{~K} ; 323,15 \mathrm{~K}$ e $348,15 \mathrm{~K}$, as quais foram controladas utilizando um termostato modificado de Berendsen V-rescale.

\section{RESULTADOS E DISCUSSÕES}

Na Figura 2 estão apresentadas, em destaque, as conformações de $180^{\circ}, 160^{\circ}, 140^{\circ}$, $110^{\circ}, 90^{\circ}$ e $80^{\circ}$ (em ordem a, b, c, d, e, f).

Figura 2 - Estruturas da amilopectina com graus de torção variados.
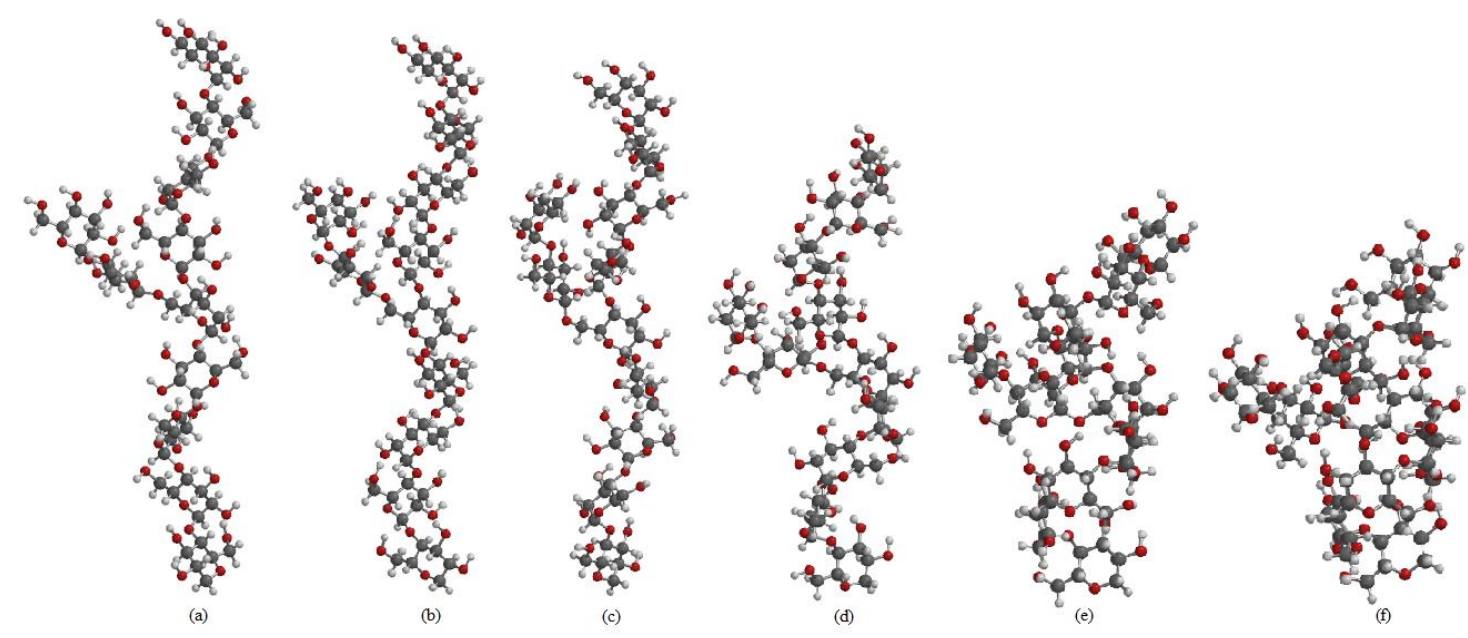

A Figura 3 demonstra uma relação entre a energia cálculada de acordo com o ângulo de torção entre os anéis. 


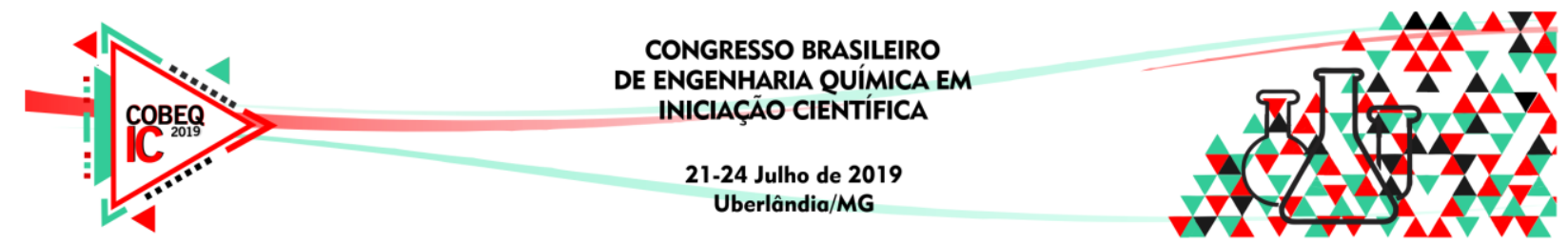

Figura 3 - Gráfico de mínimos de energia vs grau de torção.

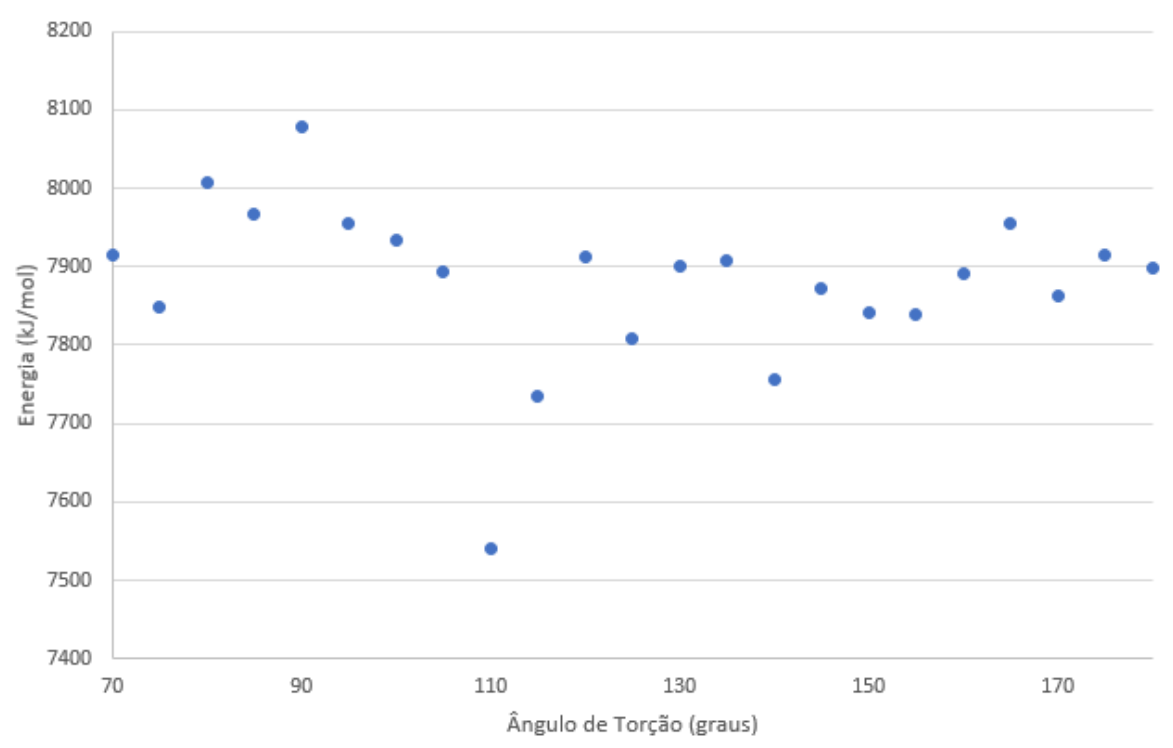

Através da análise da Figura 3 é possível determinar que, a melhor conformação para a amilopectina é a com $110^{\circ}$ de torção, isto é, ela obteve o menor valor de energia entre todas as testadas, sendo este valor obtido de $7.541,25 \mathrm{~kJ} / \mathrm{mol}$.

Após a otimização da amilopectina de $110^{\circ}$ através do servidor Automated Topology Builder (ATB), realizou-se a solvatação, como a Figura 4 demonstra.

Figura 4 - Caixa de solvatação para dinâmica molecular.

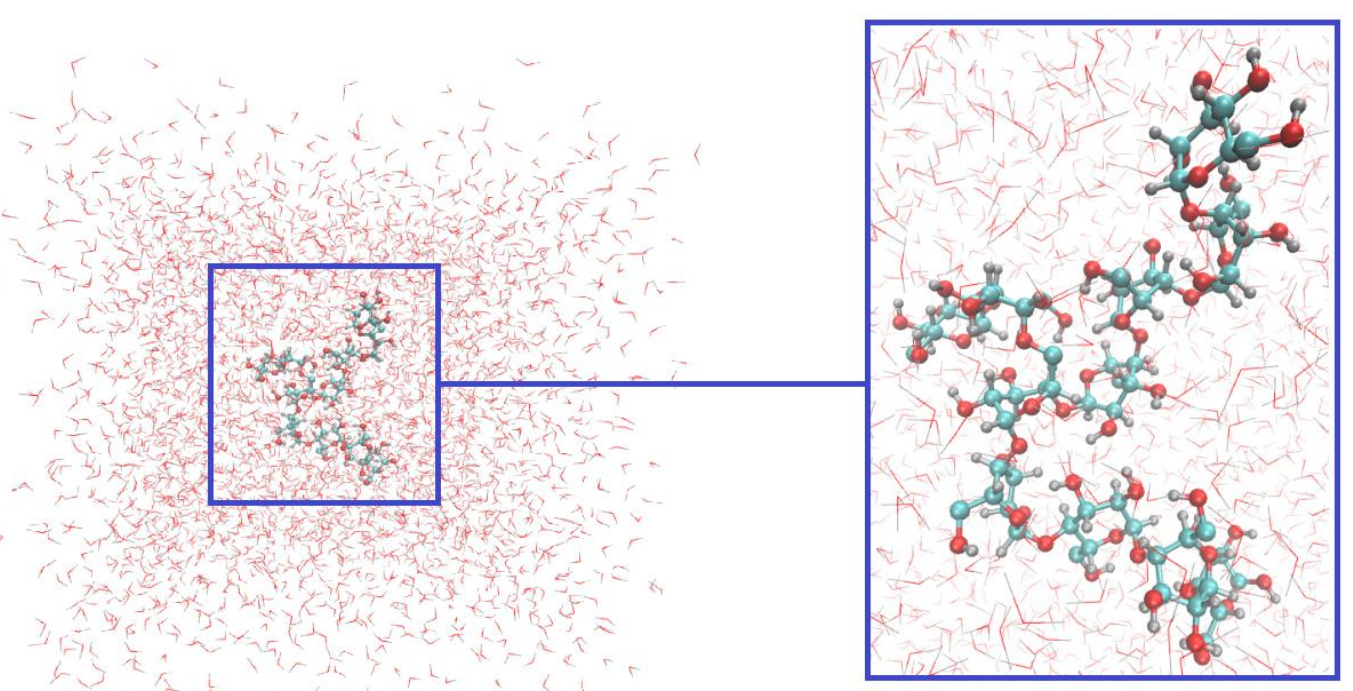

A Figura 5 demonstra os gráficos de energia cinética (a) e de energia potencial (b) para as conformações nas três temperaturas estudadas. 


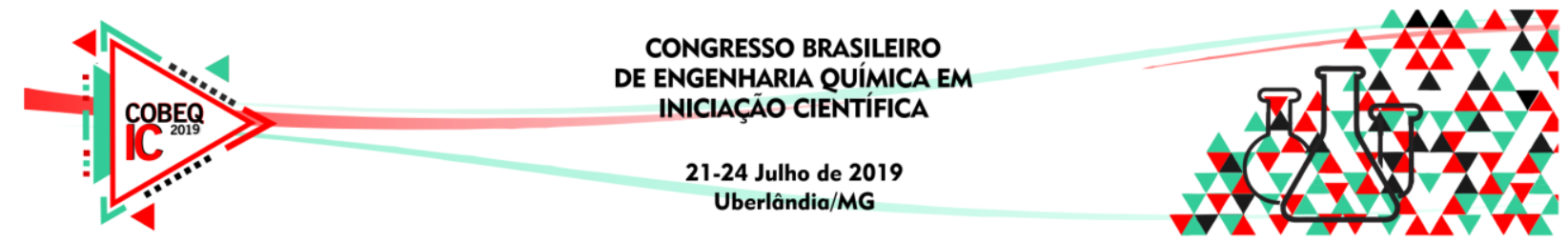

Figura 5 - (a) Energia cinética, (b) Energia potencial dos sistemas.
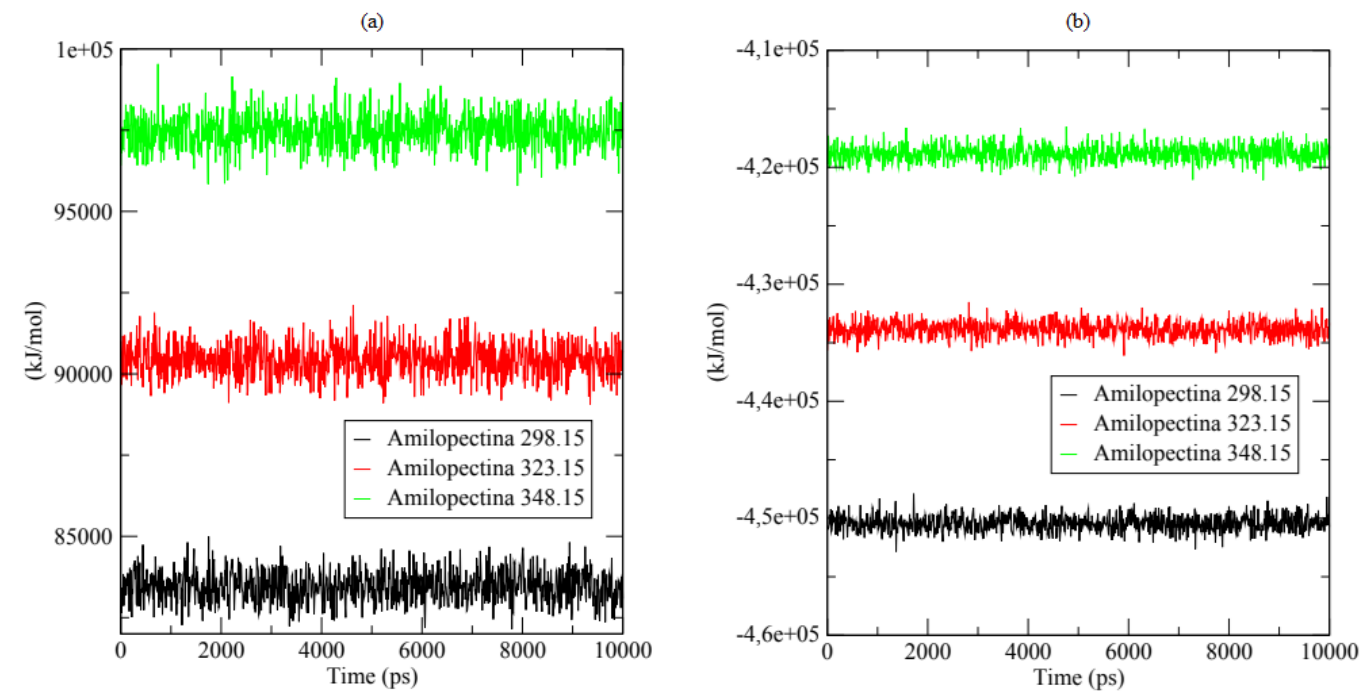

Analisando a Figura 5, é possível inferir que o sistema está de acordo com a primeira lei da termodinâmica, onde o aumento da temperatura reflete no aumento da energia cinética, devido ao aumento do movimento molecular. Para a energia potencial, as mudanças ocorrem devido a mudança em suas configurações relativas.

A Figura 6 demonstra os gráficos de energia total do sistema no decorrer da dinâmica molecular, com tempo total de 10 nanosegundos.

Figura 6 - Energia total do sistema nas três temperaturas de estudo.

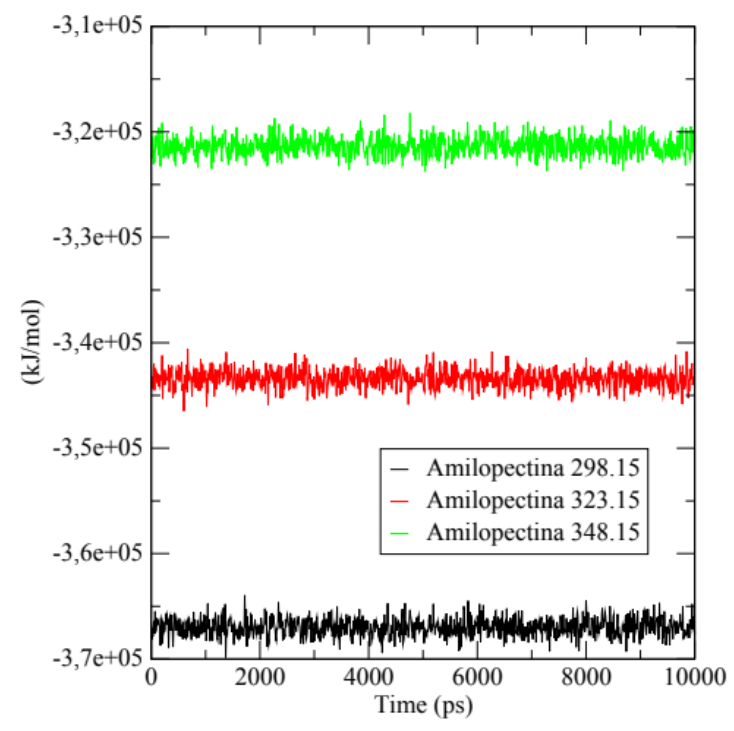

Pode-se afirmar que, através da análise da Figura 6, a energia total do sistema encontrase em uma faixa constante. A conservação da energia total demonstra que o processo de simulação de dinâmica molecular foi realizado de forma correta, como é corroborado por (Picinin, 2007). 


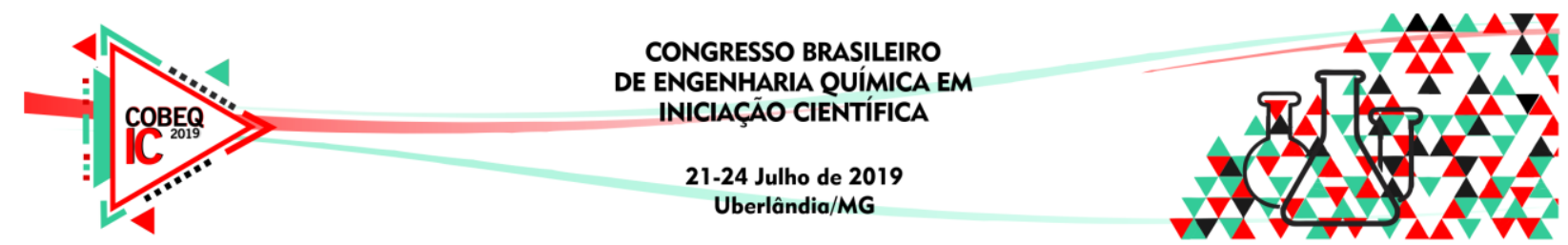

\section{CONCLUSÃO}

O estudo demonstra que para torções variáveis de $180^{\circ}$ à $70^{\circ}$, a melhor conformação, ou seja, a qual obteve o menor resultado de energia estrutural foi a amilopectina de $110^{\circ}$. Demonstrou-se também que, a variação de temperatura do sistema tem efeito direto nos valores cálculados de energia obtidos no decorrer da simulação por dinâmica molecular. Pode-se afirmar que os efeitos de temperatura estão de acordo com as leis da termodinâmica, principalmente a primeira lei. Onde o aumento da temperatura ocasionou um aumento relevante na energia cinética do sistema, bem como na energia potencial.

Os resultados obtidos demonstram que a simulação de dinâmica molecular foi realizada com sucesso, bem como os procedimentos de minimização de energia, equilíbrios NVT e NPT.

\section{REFERÊNCIAS}

BERENDSEN, H.; POSTMA, J. P.; VAN GUNSTEREN, W. F.; HERMANS, J. Interaction models for water in relation to protein hydration intermolecular forces. Netherlands: Springer, p. 331-342, 1981.

LAJOLO, F. M.; MENEZES, E. W. Carbohidratos en alimentos regionals Iberoamericanos. São Paulo: Universidade de São Paulo, 648p, 2006.

LIPORACCI, J. S. N.; MALI, S.; GROSSMANN, M. V. E. Efeito do método de extração na composição química e nas propriedades funcionais do amido de inhame (Discorea alata). Semina: Ciências Agrárias., v. 26, núm. 3, p. 345-352, 2005.

LOURDIN, D.; COIGNARD, L.; BIZOT, H.; COLONNA, P. Influence of equilibrium relative humidity and plasticizer concentration on the water content and glass transition of starch materials. Polymer, v. 38, p. 5401-5406, 1997.

PICININ, A. Simulações por dinâmica molecular aplicadas ao estudo de defeitos em cristais coloidais bidimensionais. Tese (doutorado em Física) -Instituto de física de São Carlos. Universidade de São Paulo, São Carlos. 2007.

ROCHA, G. O.; FARIAS, M. G.; CARVALHO, C. W. P.; ASCHERI, J. L. R.; GALDEANO, M. C. Filmes compostos biodegradáveis a base de amido de mandioca e proteína de soja. Polímeros, v. 24, núm. 5, p. 587-595, 2014.

SCHMID, N.; EICHENBERGER, A. P.; CHOUTKO, A.; RINIKER, S.; WINGER, M.; MARK, A. E.; VAN GUSTEREN, W. F. Definitionand testing of the GROMOS forcefield versions 54A7 and 54B7. Eur. Biophys, J. 40, 843-85610.1007/s00249-011-07009, 2011.

THIRÉ, R. M. S. M.; SIMÃO, R. A.; ARAÚJO, P. J. G.; ACHETE, C. A. Redução da hidrofilicidade de filmes biodegradáveis à base de amido por meio de polimerização por plasma. Polímeros: Ciência e Tecnologia, v.14, n. 1, p. 57-62, 2004. 